\section{SOI: $1.1 /$ TAS DOI: $10.15863 /$ TAS International Scientific Journal Theoretical \& Applied Science}

p-ISSN: 2308-4944 (print) e-ISSN: 2409-0085 (online)

Year: 2015 Issue: 12 Volume: 32

Published: $30.12 .2015 \quad$ http://T-Science.org

SECTION 8. Architecture and construction.
Aimahambet Abutalipovich Sagyndykov

Doctor of technical sciences,

Professor of the department «Building materials and constructions»»

M.Kh. Dulaty Taraz State University, Kazakhstan

Zhanna Bazartayevna Alimbayeva Senior lecturer of the department «Architecture and construction production» M.Kh. Dulaty Taraz State University, Kazakhstan

Akpan Turabaevich Kyrgyzbaev Candidate of technical sciences, associate professor of the Department of «Building materials and constructions»

M.Kh. Dulaty Taraz State University, Kazakhstan

Bauyrzhan Zharkynbekovich Manapbayev Candidate of technical sciences, associate professor of the Department of «Oil and gas engineering»

M.Kh. Dulaty Taraz State University, Kazakhstan jiboj@mail.ru

\title{
STUDY OF STRENGTH CHARACTERISTICS OF HIGHLY POROUS CERAMICS
}

Abstract: The article presents the results of selection of optimum extinguishing efficiency of the formulations based on highly porous ceramics of an optimum loam, ash and cullet. Gazkeramik installed mineral composition using $x$-ray phase analysis.

Key words: ceramics, strength, mathematical modelling.

Language: Russian

Citation: Sagyndykov AA, Alimbayeva ZB, Kyrgyzbaev AT, Manapbayev BZ (2015) STUDY OF STRENGTH CHARACTERISTICS OF HIGHLY POROUS CERAMICS. ISJ Theoretical \& Applied Science 12 (32): 118-121.

Soi: http://s-o-i.org/1.1/TAS-12-32-23 Doi: crossef http://dx.doi.org/10.15863/TAS.2015.12.32.23

\section{ИССЛЕДОВАНИЕ ПРОЧНОСТНЫХ ХАРАКТЕРИСТИК ВЫСОКОПОРИСТОЙ КЕРАМИКИ}

Аннотация: В статье приведены результаты подбора оптимальных составов высокопористой керамики на основе суглинка, стеклобоя и зольл. С помощью рентгенофазового анализа установлен минеральный состав газокерамики.

Ключевые слова: керамика, прочность, математическое моделирование.

В настоящее время наиболее широкое распространение в строительстве получили керамические пористые материалы. Этот материал является, прежде всего, конструкционно-теплоизоляционным прочностью до 2-3 МПа, и целесообразно повысить его прочность до 5-7 МПа [1-3]. Повышения прочности материала требует дальнейших физико-химических исследований по изучению влияния основных факторов на его прочностные свойства.

Как показали исследования структурообразования, физико-механических и технологических свойств высокопористой керамики на основе суглинка и золы они характеризуются относительно высокой температурой обжига порядка $1000-1050^{\circ} \mathrm{C}$. Для снижения температуры обжига была использована добавка стеклобоя.

С целью определения оптимального состава газокерамики нами использованы методы математического моделирования и оптимизации.

Решение данной задачи было получено с использованных специально разработанных программ для персонального компьютера (система Mathcad-14 [2-5] и выполнено в два этапа:

1. получение адекватных исследуемому объекту математических модели, описывающей 
влияние состава исходной смеси на выбранные целевые показатели качества получаемого изделия;

2. поиск оптимального состава исходных смесей с использованием полученных математических моделей.

В качестве входных переменных (факторов) использованы следующие параметры состава исходной смеси:
$\mathrm{X}_{1}-$ суглинок $(\mathrm{C \Gamma})$;

$\mathrm{X}_{2}-$ стеклобой $($ СБ ;

$\mathrm{X}_{3}$ - зола (ЗЛ);

На основе предварительного анализа объекта исследования выбраны следующие диапазоны изменения факторов (входов X) (см. табл.1).

\section{Таблица 1}

Диапазоны изменения режимных параметров (входов).

\begin{tabular}{|l|c|c|c|}
\hline \multicolumn{1}{|c|}{ Факторы } & $\mathrm{X}_{1}$ & $\mathrm{X}_{2}$ & $\mathrm{X}_{3}$ \\
\hline Нижний уровень $(-1)$ & 50 & 3 & 20 \\
\hline Верхний уровень $(+1)$ & 70 & 7 & 30 \\
\hline Нулевой уровень $(0)$ (середина плана) & 60 & 5 & 25 \\
\hline Интервал варьирования $(\Delta \mathrm{X})$ & 10 & 2 & 5 \\
\hline Плечо $-\alpha(\alpha=1,682)$ (дополнительные точки) & 43,18 & 1,636 & 16,59 \\
\hline Плечо + $\alpha$ (дополнительные точки) & 76,82 & 8,364 & 33,41 \\
\hline
\end{tabular}

Для математической обработки результатов проведенных экспериментов для поиска оптимального состава исходной смеси нами использован метод нелинейного программирования на основе т.н. квазиньютоновского алгоритма [4-8], реализованного в системе Mathcad-14 (результаты приведены в табл. 2).

План, результаты проведенных эксперимента и имитационного моделирования, полученные значения коэффициентов математической модели в безразмерном (кодированном) и натуральном масштабах, ошибки аппроксимации, а также значения критерия адекватности R-квадрат сведены нами в табл.2. Там же приведены найденные оптимальные составы исходной смеси, обеспечивающие максимальную и минимальную прочность изделия.

Таблица 2

План и результаты проведенных экспериментов для выхода $\mathrm{Y}=\mathrm{Y}_{1}$ (прочность изделия).

\begin{tabular}{|c|c|c|c|c|c|c|c|c|c|}
\hline \multirow[b]{2}{*}{$\begin{array}{c}\text { № } \\
\text { опыта }\end{array}$} & \multicolumn{3}{|c|}{ Входные переменные } & \multicolumn{2}{|c|}{ Выход } & \multicolumn{2}{|c|}{ Погрешность (ошибка) } & \multirow{2}{*}{\multicolumn{2}{|c|}{$\begin{array}{c}\text { Коэффициенты } \\
\text { математической модели в } \\
\text { натуральном масштабе } \\
\end{array}$}} \\
\hline & $\mathrm{X}_{1}$ & $\mathrm{X}_{2}$ & $\mathrm{X}_{3}$ & Үэксп. & Үрасч. & \begin{tabular}{|c|} 
абсолютна \\
я
\end{tabular} & относительная \% & & \\
\hline 1 & 50,00 & 3,00 & 20,00 & 7,100 & 7,0871 & 0,0129 & 0,1819 & № & Значение \\
\hline 2 & 70,00 & 3,00 & 20,00 & 7,400 & 7,2142 & 0,1858 & 2,5106 & B1 & 37,8057 \\
\hline 3 & 50,00 & 7,00 & 20,00 & 3,800 & 3,8366 & $-0,0366$ & $-0,9632$ & $\mathrm{~B} 2$ & $-0,1810$ \\
\hline 4 & 70,00 & 7,00 & 20,00 & 3,600 & 3,7137 & $-0,1137$ & $-3,1593$ & B3 & $-2,7471$ \\
\hline 5 & 50,00 & 3,00 & 30,00 & 6,100 & 5,8684 & 0,2316 & 3,7974 & B4 & $-1,5368$ \\
\hline 6 & 70,00 & 3,00 & 30,00 & 6,400 & 6,2455 & 0,1545 & 2,4142 & B5 & 0,0014 \\
\hline 7 & 50,00 & 7,00 & 30,00 & 3,100 & 3,1679 & $-0,0679$ & $-2,1894$ & B6 & 0,1816 \\
\hline 8 & 70,00 & 7,00 & 30,00 & 3,400 & 3,2950 & 0,1050 & 3,0881 & B7 & 0,0262 \\
\hline 9 & 43,18 & 5,00 & 25,00 & 3,800 & 3,8264 & $-0,0264$ & $-0,6957$ & B8 & $-0,0031$ \\
\hline 10 & 76,82 & 5,00 & 25,00 & 3,900 & 4,0403 & $-0,1403$ & $-3,5967$ & B9 & 0,0012 \\
\hline 11 & 60,00 & 1,64 & 25,00 & 7,900 & 8,1909 & $-0,2909$ & $-3,6818$ & B10 & 0,0137 \\
\hline 12 & 60,00 & 8,36 & 25,00 & 3,100 & 2,9758 & 0,1242 & 4,0050 & & \\
\hline 13 & 60,00 & 5,00 & 16,59 & 6,100 & 6,0719 & 0,0281 & 0,4606 & \multirow{3}{*}{\multicolumn{2}{|c|}{$\begin{array}{c}\text { Коэффициенты } \\
\text { математической модели в } \\
\text { безразмерном масштабе }\end{array}$}} \\
\hline 14 & 60,00 & 5,00 & 33,41 & 4,500 & 4,6948 & $-0,1948$ & $-4,3289$ & & \\
\hline 15 & 60,00 & 5,00 & 25,00 & 3,520 & 3,5286 & $-0,0086$ & $-0,2439$ & & \\
\hline 16 & 60,00 & 5,00 & 25,00 & 3,540 & 3,5286 & 0,0114 & 0,3224 & № & Значение \\
\hline 17 & 60,00 & 5,00 & 25,00 & 3,490 & 3,5286 & $-0,0386$ & $-1,1056$ & B1 & 3,5172 \\
\hline
\end{tabular}




\begin{tabular}{|c|c|c|c|c|c|c|}
\hline Impact Factor: & $\begin{array}{l}\text { ISRA (India) } \\
\text { ISI (Dubai, UAF } \\
\text { GIF (Australia) } \\
\text { JIF }\end{array}$ & $\begin{array}{l}=1.344 \\
=0.829 \\
=0.564 \\
=1.500\end{array}$ & $\begin{array}{l}\text { SIS (USA) = } \\
\text { PUHЦ (Russia) } \\
\text { ESJI (KZ) = } \\
\text { SJIF (Morocco) }\end{array}$ & $\begin{array}{l}=0.912 \\
=0.179 \\
=1.042 \\
=2.031\end{array}$ & $\begin{array}{l}\text { ICV (Poland) } \\
\text { PIF (India) }\end{array}$ & $\begin{array}{l}=6.630 \\
=1.940\end{array}$ \\
\hline
\end{tabular}

\begin{tabular}{|c|c|c|c|c|c|c|c|c|c|}
\hline 18 & 60,00 & 5,00 & 25,00 & 3,550 & 3,5286 & 0,0214 & 0,6032 & $\mathrm{~B} 2$ & 0,0389 \\
\hline 19 & 60,00 & 5,00 & 25,00 & 3,500 & 3,5286 & $-0,0286$ & $-0,8167$ & B3 & $-1,5133$ \\
\hline 20 & 60,00 & 5,00 & 25,00 & 3,600 & 3,5286 & 0,0714 & 1,9837 & B4 & $-0,5941$ \\
\hline \multicolumn{6}{|c|}{ Суммарная ошибка $=$} & $-1,680 \mathrm{E}-10$ & $-1,4141$ & B5 & 0,1823 \\
\hline \multicolumn{6}{|c|}{ Среднее значение ошибки $=$} & $-8,402 \mathrm{E}-12$ & $-0,0707$ & B6 & 0,7478 \\
\hline \multicolumn{6}{|c|}{ Значение критерия R квадрат = } & \multicolumn{2}{|c|}{0,9936} & B7 & 0,4651 \\
\hline \multicolumn{6}{|c|}{ Найденный режим с минимальным значением Ү } & & & B8 & $-0,0625$ \\
\hline & Х1мин & Х2мин & Х3мин & \multirow{2}{*}{\multicolumn{2}{|c|}{ Үмин }} & & & B9 & 0,0625 \\
\hline & 59,617 & 3,09249 & 26,022 & & & & & B10 & 0,1375 \\
\hline \multicolumn{6}{|c|}{$\begin{array}{c}\text { Найденный режим с максимальным значением } \\
\text { Y }\end{array}$} & & & & \\
\hline & 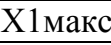 & X2макс & Х3макс & \multirow{2}{*}{\multicolumn{2}{|c|}{$\begin{array}{l}\text { Үмкс } \\
1.42\end{array}$}} & & & & \\
\hline & 43,18 & 6,636 & 16,59 & & & & & & \\
\hline
\end{tabular}

Проверка значимости по критерию Стьюдента [4] показала, что все 10 найденных коэффициентов математической модели (1) значимы.

Проверка по критерию Фишера [4], показало, что уравнение (1) адекватно описывает результаты эксперимента (расчетное значение критерия Фишера равное 3,0 , что меньше критического равного 5,1$)$.
На рисунке 1 приведены графики зависимости изменения ошибки аппроксимации для каждого опыта, видно, что ошибка небольшая, в таблице 2 приведены численные значения ошибок.

Получено значение $\mathbf{R}^{2}=\mathbf{0 , 9 9 3 6}$, т.е. его значение достаточно близко к 1 , что дополнительно подтверждает адекватность полученной математической модели (1).

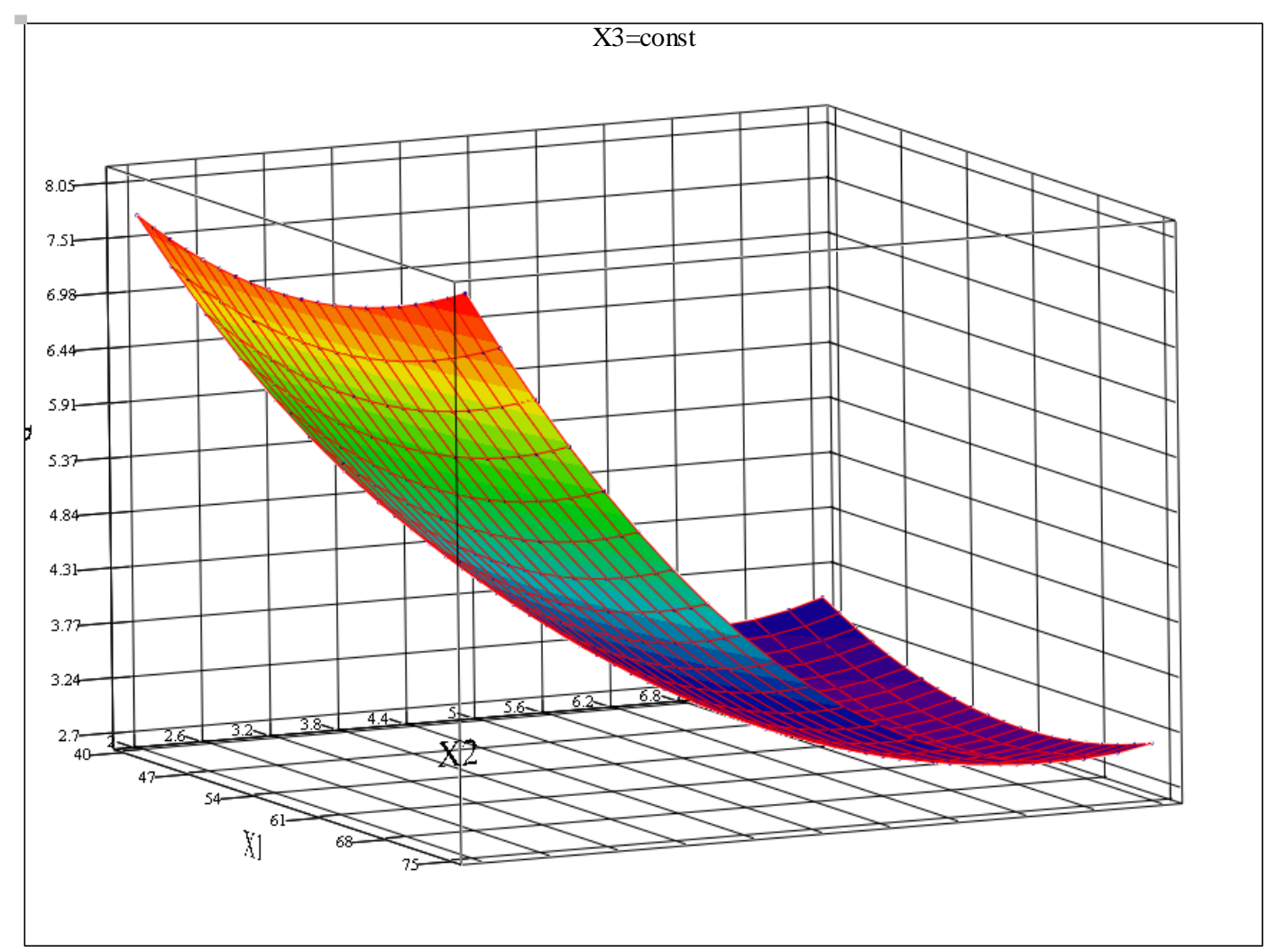

Y3

Рисунок 1. Трехмерный график функции Y при фиксированном значении $\mathrm{X}_{3}$ со значением в середине плана $\left(X_{3}=25\right)$.

Полученная адекватная математическая модель позволяет осуществить поиск оптимальных режимных параметров процесса, обеспечивающих минимальную прочность изделия при поиске оптимума в диапазоне ограничений поиска оптимума от $-\alpha$ до $+\alpha$ : 


\begin{tabular}{l|lrl|l|ll} 
& ISRA (India) & $=\mathbf{1 . 3 4 4}$ & SIS (USA) & $=\mathbf{0 . 9 1 2}$ & ICV (Poland) & $=\mathbf{6 . 6 3 0}$ \\
Impact Factor: & ISI (Dubai, UAE) $=\mathbf{0 . 8 2 9}$ & PUHL (Russia) $=\mathbf{0 . 1 7 9}$ & PIF (India) & $=\mathbf{1 . 9 4 0}$ \\
& GIF (Australia) & $\mathbf{0 . 5 6 4}$ & ESJI (KZ) & $=\mathbf{1 . 0 4 2}$ & & \\
& JIF & $\mathbf{1 . 5 0 0}$ & SJIF (Morocco) $=\mathbf{2 . 0 3 1}$ & &
\end{tabular}

\begin{tabular}{|c|c|c|c|}
\hline $\mathrm{X}_{1 \text { макс }}$ & $\mathrm{X}_{2 \text { макс }}$ & $\mathrm{X}_{3 \text { макс }}$ & Үмакс \\
\hline 43,18 & 6,636 & 16,59 & 11,42 \\
\hline
\end{tabular}

и при поиске оптимума в диапазоне ограничений поиска оптимума от- -1 до +1 :

\begin{tabular}{|c|c|c|c|}
\hline $\mathrm{X}_{1}$ макс & $\mathrm{X}_{2}$ макс & $\mathrm{X}_{3}$ макс & Үмакс \\
\hline 50 & 5 & 20 & 7,09 \\
\hline
\end{tabular}

Проведя имитационное моделирование с использованием уравнения (1) и найденных параметров модели нами получены графики зависимостей прочность изделия от значений режимных параметров, показанные на рис.1.Наиболее значимыми факторами повышающие прочность являются содержание суглинка и стеклобоя. С увеличением количества золы в композиций водопоглощение обожженных образцов увеличивается. Тем не менее, можно

выделить предельные дозировки зол в композиции, при которых обеспечивается получение образцов с высоким эксплуатационными свойствами. Из графика изменения видно, что этот предел равен $15-20 \%$. Наименьшей прочностью 3-4 МПа имеют составы содержащие, мас. суглинок 85-90, зола25-30, стеклобой-5, а наибольшей составы 7-11 МПа содержащие суглинок 75-80, зола -15-20, стеклобой 5-7. Оптимальный состав высокопористой керамики: суглинок 75-80; стеклобой 5-7; зола 15-20. Физико-механические свойства: прочность при сжатии 6-7,8 МПа; плотность: 650-750 кг/м; водопоглощение 25 $30 \%$; теплопроводность 0,1-0,2 Вт/мС. Согласно рентгенофазовому анализу регистрируются кристаллические фазы кварца, анортита, волластонита способствующие повышению прочности.

\section{References:}

1. Ezerskiy VA, Korovyakov VF, Krolevetskiy DV (2006) Tekhnologiya penokeramicheskikh stenovykh i teploizolyatsionnykh izdeliy // Krovel'nye i izolyatsionnye materialy. - 2006, № 1. - pp. 68-69.

2. Anderson MA (1984) Nek Low- cost PFA brickmaking procese // Ach Techn' 84:2 - nd Int. Conf. Ach TchnoL and Market. London. Sept. 16-21.-1984.- pp. 563-567.

3. Salmang K (1998) Die physikalischen und chemishen Crundlagen der Keramik / H. Salmang, H. Schoize. - Berlin: Springer Verl., 1998. - 450 p.

4. Akhnazarova SL, Kafarov VV (1985) Metody optimizatsii eksperimenta $\mathrm{V}$ khimicheskoy tekhnologii: Uchebnoe posobie dlya vuzov. - 2e izdanie, pererab. i dopolnennoe. -Moscow: Vysshaya shkola, 1985. -327 p.

5. Abraham B, Ledolter J (1983) Statistical methods for forecasting. New York: Wiley.

6. Ochkov V (2007) Mathcad 14 dlya studentov, inzhenerov i konstruktorov. - SPb.: BHVPeterburg, 2007.

7. Khimmel'blau D (1985) Prikladnoe nelineynoe programmirovanie -Moscow: Mir, 1985. -554 p.

8. Chernorutskiy IG (2004) Metody optimizatsii v teorii upravleniya. Uchebnoe posobie / I. G. Chernorutskiy. - SPb.: Piter, 2004. - 256 p. 